\title{
Network pharmacology and UPLC-Q-TOF/MS studies on the anti-arthritic mechanism of Pterocephalus hookeri
}

\author{
Ce Tang ${ }^{1}$, Hai-Jiao Li ${ }^{1}$, Gang Fan ${ }^{1}$, Ting-Ting Kuang ${ }^{1}$, Xian-Li Meng ${ }^{1}$, Zhong-Mei \\ Zou $^{2}$, Yi Zhang ${ }^{1 *}$ \\ ${ }^{1}$ College of Ethnic Medicine, Chengdu University of Traditional Chinese Medicine, Chengdu 611137, ${ }^{2}$ Institute of Medicinal \\ Plant Development, Chinese Academy of Medical Sciences and Peking Union Medical College, Beijing 100193, China
}

*For correspondence: Email: 1175332408@qq.com; Tel: +862861800074

Sent for review: 17 February 2018

Revised accepted: 26 May 2018

\begin{abstract}
Purpose: To investigate the mechanism underlying the anti-arthritic properties of Pterocephalus hookeri used for treatment of rheumatoid arthritis (RA).

Methods: Aqueous methanol extract of P. hookeri was analyzed using UPLC-Q-TOF/MS, a Waters Acquity UPLCR BEH C18 column $(2.1 \times 100 \mathrm{~mm}, 1.7 \mu \mathrm{m})$ and gradient elution with acetonitrile-formic acid-water. Targets and related pathways were predicted by PharmMapper database and Molecule Annotation System, respectively. The network was built with Cytoscape software.

Results: Forty compounds were identified, comprising 17 iridoid glycosides, 7 phenolic acids, 13 triterpenes, and 3 other compounds. A total of 38 targets and 44 pathways associated with RA were obtained. These involved mainly MAPK signaling pathway, adherens junction, and colorectal cancer.

Conclusion: These results from network pharmacology suggest that $P$. hookeri exerts therapeutic effect on RA via multiple components, multiple targets and multiple pathways.
\end{abstract}

Keywords: Pterocephalus hookeri, Rheumatoid arthritis, UPLC-Q-TOF/MS, Chemical composition, Network pharmacology

\begin{abstract}
This is an Open Access article that uses a funding model which does not charge readers or their institutions for access and distributed under the terms of the Creative Commons Attribution License (http://creativecommons.org/licenses/by/4.0) and the Budapest Open Access Initiative (http://www.budapestopenaccessinitiative.org/read), which permit unrestricted use, distribution, and reproduction in any medium, provided the original work is properly credited.
\end{abstract}

Tropical Journal of Pharmaceutical Research is indexed by Science Citation Index (SciSearch), Scopus, International Pharmaceutical Abstract, Chemical Abstracts, Embase, Index Copernicus, EBSCO, African Index Medicus, JournalSeek, Journal Citation Reports/Science Edition, Directory of Open Access Journals (DOAJ), African Journal Online, Bioline International, Open-J-Gate and Pharmacy Abstracts

\section{INTRODUCTION}

Rheumatoid arthritis (RA) is a systemic autoimmune disease which occurs in three stages: chronic, progressive, and aggressive arthritis, eventually leading to joint deformity and loss of function. Thus, RA has a high frequency of disability [1]. In Tibetan medicine, RA is called "zhen bu" disease [2], and available data show that the treatment efficacy of Tibetan medicine for RA is up to $94.6 \%[3,4]$.
Previous studies have confirmed that the major chemical components of $P$. hookeri include iridoid glycosides, triterpenoid saponins, and phenolic acids $[5,6]$. A survey of ancient literature revealed that $P$. hookeri was frequently used in the treatment of RA [7]. Some recent studies have shown that aqueous and ethanolic extracts of $P$. hookeri have anti-inflammatory, anti-RA, and analgesic effects $[8,9]$. Although there are several studies on $P$. hookeri, its mechanism of 
action on RA has not received much attention.

Recently, network-based analyses have emerged as powerful tools for elucidating the multiple and active components of extracts, as well as their mechanisms of action [10]. A new approach is provided by network pharmacology for the study of activities of multiple components and pharmacological mechanisms in traditional medicine.

The purpose of the research was to use network pharmacology and UPLC-Q-TOF/MS to unravel the active ingredients of $P$. hookeri, their targets, and the mechanism involved in the anti-RA effect of the plant.

\section{EXPERIMENTAL}

\section{Materials and reagents}

Acetonitrile and formic acid (HPLC grade) were supplied by Merck (Darmstadt, Germany) and Fluka (Buchs, Steinheim, Germany), respectively. Distilled water used for UPLC-QTOF/MS was acquired using the Milli-Q system (Millipore, France), while $P$. hookeri was purchased from Ganzi Tibetan Hospital in Sichuan, China. The standards ( $98 \%$ purity) of loganic acid (3), chlorogenic acid (4), sweroside (9), loganin (10), 6-apiofuranosylsweroside (12), 3,4-dicaffeoylquinic acid (15), 3,5-dicaffeoylquinic acid (16), 4,5-dicaffeoylquinic acid (18), ursolic acid (39), and oleanolic acid (40) were supplied by the National Institute for Food and Drug Control (Beijing, China) or Chengdu Must BioTechnology Co., Ltd. (Chengdu, Ching). Sylvestroside I (17), cantleyoside (19), triplostoside A (25), dipsanosides B (26), and dipsanosides A (27) were acquired from $P$. hookeri by in previous studies $[11,12]$.

\section{Sample preparation}

Powered $P$. hookeri $(2.0 \mathrm{~g})$ was accurately weighed and added to $50 \mathrm{~mL}$ of $70 \%$ aqueous methanol. The mixture was subjected to ultrasonic extraction for $30 \mathrm{~min}$, and filtered. The residue was washed with a small amount of $70 \%$ aqueous methanol, and the combined filtrate was concentrated and dissolved in the same solvent. The concentrated solution was transferred to a $10 \mathrm{~mL}$ bottle, and $70 \%$ methanol was added to the mark. The supernatant was centrifuged at $14000 \mathrm{rpm}$ for $15 \mathrm{~min}$ and filtered through a 0.22 $\mu \mathrm{m}$ microporous membrane.

\section{UPLC chromatography}

UPLC was performed in a $100 \mathrm{~mm} \times 2.1 \mathrm{~mm}, 1.7$ $\mu \mathrm{m}$ Waters Acquity UPLC ${ }^{\mathrm{R}}$ BEH C18 column (Waters Corp., Milford, USA). The mobile phase was composed of acetonitrile $(A)$, and water $(B)$ each containing $0.1 \%$ formic acid. The linear gradient program was performed as follows: 5 $12 \%$ B for 0 - $7 \mathrm{~min} ; 12$ - $17 \%$ B for 7 - $8 \mathrm{~min}$; $17 \%$ B for 8 - 13 min; $17-25 \%$ B for $13-20$ min; 25 - $46 \%$ B for 20 - 23 min; $46 \%$ B for 23 26 min; 46 - $60 \%$ B for 23 - 32 min; 60 - $95 \%$ B for 32 - $35 \mathrm{~min}$, and $95 \% \mathrm{~B}$ for 35 - $36 \mathrm{~min}$. Flow rate, $0.4 \mathrm{~mL} / \mathrm{min}$; injection volume, $2 \mu \mathrm{L}$; column temperature, $40{ }^{\circ} \mathrm{C}$.

\section{Mass spectrometry}

Waters SYNAPT G2HDMS system of ion source was used for electrospray ionization (ESI). Scanning was done at positive $(E S I+)$ and negative (ESI-) ion modes, with nitrogen as atomization and conical gas. The source temperature and cone gas flow rate were $100{ }^{\circ} \mathrm{C}$ and $40 \mathrm{~L} / \mathrm{h}$, respectively. Desolvation temperature and gas flow rate were $350{ }^{\circ} \mathrm{C}$ and $800 \mathrm{~L} / \mathrm{h}$, respectively. Other MS conditions used were sampling cone voltage of $40 \mathrm{~V}$, extraction cone voltage of $4 \mathrm{~V}$, capillary voltages of $3.0 \mathrm{kV}$ (ESI+) and $2.5 \mathrm{kV}$ (ESI-), scan time and inter scan time (0.3 s and $0.02 \mathrm{~s}$, respectively), and mass-to-charge ratio, $\mathrm{m} / \mathrm{z}$ of 50 - 1700. Leucineenkephalin $(0.5 \mu \mathrm{g} / \mathrm{mL})$ at a flow velocity of 5 $\mu \mathrm{L} / \mathrm{min}$, was used for calibration of mass number $(\mathrm{m} / z 556.2771$ for $\mathrm{ESI}+$, and $\mathrm{m} / \mathrm{z} 554.2615$ for ESI-).

\section{Prediction and screening of targets}

ChemBio Office 2014 software was used to draw the structures of the compounds. These were converted to 3D forms with ChemBio3D ultra software, and stored in mol2 format. Then, in order to predict the potential target, chemical components were imported into the PharmMapper website (http://lilab.ecust.edu.cn/pharmmapper/) for potential target prediction analysis. The first 10 targets of each compound were selected for follow-up study.

\section{Prediction and screening of pathways}

The targets obtained were introduced into the Bio database (http://bioinfo.capitalbio. com/mas3/) and then screened for pathways that met the criterion of $p<0.01$.

\section{Construction of network}

Cytoscape software was used to construct compound-target-pathway networks with 
chemical constituents, predicted targets and pathways.

\section{RESULTS}

\section{Compositions of $P$. hookeri}

Data on the ESI+ and ESI- modes are shown in Figure 1. It was found that ESI- mode provided a neater MS fragmentation and sharper peak shapes than $\mathrm{ESI}+$. However, it was easier to find the quasi-molecular ion peaks of compounds with combination of positive and negative ions. Using relative retention times, exact masses, MS fragments, standards, and references, 40 compounds were identified or elucidated with the information shown in Figure 1, Figure 2, Table 1.

Compounds of iridoid glycosides have variety of activities, such as anti-inflammatory [8], anti-RA [2], and the anti-tumor [24] activities. A total of 17 $(1,3,7-12,17,19-21,23-27)$ compounds were identified at the same time in ESI+ and ESImodes. A glucose unit (Glc, $162 \mathrm{Da})$ was a representative fragment during a neutral loss scan of all iridoid glycosides. Taking peak $\mathbf{3}$ as an example, the quasi-molecular ions $[2 \mathrm{M}+\mathrm{Na}-$ $2 \mathrm{H}^{-}$at $\mathrm{m} / \mathrm{z} 773.2469$, [2M-H] at $\mathrm{m} / \mathrm{z} 751.2656$, and $[\mathrm{M}-\mathrm{H}]^{-}$at $\mathrm{m} / \mathrm{z} 375.1281$, indicated that the formula of the compound was $\mathrm{C}_{16} \mathrm{H}_{24} \mathrm{O}_{10}$. Figure $3 \mathrm{~A}$ indicates that $[\mathrm{M}-\mathrm{H}]^{-}$of peak 3 got the ions at $\mathrm{m} / \mathrm{z} 213.0759$ and 169.0859 by losing $162 \mathrm{Da}$ (Glc) and $44 \mathrm{Da}\left(\mathrm{CO}_{2}\right)$. Besides, the ions at $\mathrm{m} / \mathrm{z}$ 113.0239 and 151.0752 were generated by the characteristic retro-Diels-Alder (RDA) cleavage loss of $\mathrm{C}_{3} \mathrm{H}_{4} \mathrm{O}\left(\begin{array}{ll}56 & \mathrm{Da}\end{array}\right)$ and $\mathrm{H}_{2} \mathrm{O}\left(\begin{array}{ll}18 \mathrm{Da}\end{array}\right)$, respectively from the fragment ion at $\mathrm{m} / \mathrm{z}$ 169.0859 [15]. As a consequence, by comparison with standard, peak $\mathbf{3}$ was confirmed as loganic acid. The proposed fragmentation pathway of loganic acid (peak 3 ) is displayed in Figure 3B.

Seven $(2,4-6,15,16,18)$ compounds were identified as phenolic acids from $P$. hookeri in the $\mathrm{ESI}+$ and ESI- modes. Peak 4 generated a [2M$\mathrm{H}]^{-}$ion at $\mathrm{m} / \mathrm{z} 707.1810,[\mathrm{M}+\mathrm{Na}-2 \mathrm{H}]^{-}$ion at $\mathrm{m} / \mathrm{z}$ 375.0866, and $[\mathrm{M}-\mathrm{H}]$ ] ion at $\mathrm{m} / \mathrm{z}$ 353.0866, indicated that the formula of the compound was $\mathrm{C}_{16} \mathrm{H}_{18} \mathrm{O}_{9}$. The $\left[\mathrm{M}-\mathrm{H}-\mathrm{C}_{9} \mathrm{H}_{6} \mathrm{O}_{3}\right]^{-}$ion at $\mathrm{m} / \mathrm{z}$ 191.0560, and [caffeic acid-H] ion at $\mathrm{m} / \mathrm{z}$ 179.0350 showed that the compound structure contained a quinic acid and caffeic acid. Comparing with standard, peak $\mathbf{4}$ was unambiguously confirmed as chlorogenic acid. Peaks 2 and 6 had uniform quasi-molecular ion at $\mathrm{m} / \mathrm{z}$ 353, which indicates that these two compounds were isomeric with $\mathbf{4}$. It was easy to distinguish peaks 2 and 6 using literature reports and their relative retention times [14]. Peaks 15,
16 , and 18 were had the same quasi-molecular and fragment ions, indicating that these three compounds were isomeric. On the basis of comparisons with standards, and literature reports, the three peaks were confirmed as 3,4dicaffeoylquinic acid, 3,5-dicaffeoylquinic acid, and 4,5-dicaffeoylquinic acid. The derivation of the fragmentation pathway of 3,5-dicaffeoylquinic acid is shown in Figure 4B.

The other major chemical constituents of $P$. hookeri were triterpenes. A triterpene molecule is an aglycone formed after the removal the removal of one or more sugar moieties from a triterpene glycoside. The most common sugar moieties in triterpenes are glucose (Glc), rhamnose (Rha), and xylose (Xyl), and they are linked to the aglycone through O-glycosidic linkages in positions 3 and 28. Therefore, the loss of $162 \mathrm{Da}(\mathrm{Glc}), 132 \mathrm{Da}$ (Rha), and $146 \mathrm{Da}$ (Xyl) in MS data are characteristic for triterpenes. The triterpenes differ in type and amount of sugar present. Thirteen triterpenes (28-40) were identified. For example, peak $\mathbf{3 5}$ exhibited ions at $\mathrm{m} / \mathrm{z} 1033.5337([\mathrm{M}+\mathrm{Na}-2 \mathrm{H}]]^{)}$) and 1011.5532 ([M$\mathrm{H}]$ ), corresponding to the molecular formula $\mathrm{C}_{52} \mathrm{H}_{84} \mathrm{O}_{19}$. The MS spectrum of peak 35 gave four major fragment ions at $m / z 865.4962([\mathrm{M}-\mathrm{H}-$ Rha] ]), 733.4523 ([M-H-Rha-Xyl]'), 587.3956 ([MH-2Rha-Xyl]), and 455.3532 ([M-H-2Rha-2Xyl]'), demonstrating the presence of two rhamnose and xylose residues. From references, peak 35 was confirmed as triploside $G$. The proposed fragmentation pathway of triploside $G$ (peak 35) is shown in Figure 5B.

Apart from iridoid glycosides, triterpenes, and phenolic acids, three other compounds $(\mathbf{1 3}, \mathbf{1 4}$, 22) were identified from $P$. hookeri. Additional information about these compounds and their chemical constitutions are shown in Table 1 and Figure 2, respectively.

\section{Network pharmacology}

A total of 38 targets were obtained by importing 40 chemical compositions predicted to be absorbable into the PharmMapper database for directional docking (Table 2). These targets were then imported into the Molecule Annotation System, which resulted 57 pathways regulated by $P$. hookeri with significant differences $(p<$ $0.05)$. Forty-four of these pathways that met the criterion of $P<0.01$ (Table 3).

Cytoscape software was used to construct a pharmacology network of $P$. hookeri to generate the correlations of chemical components, targets, 

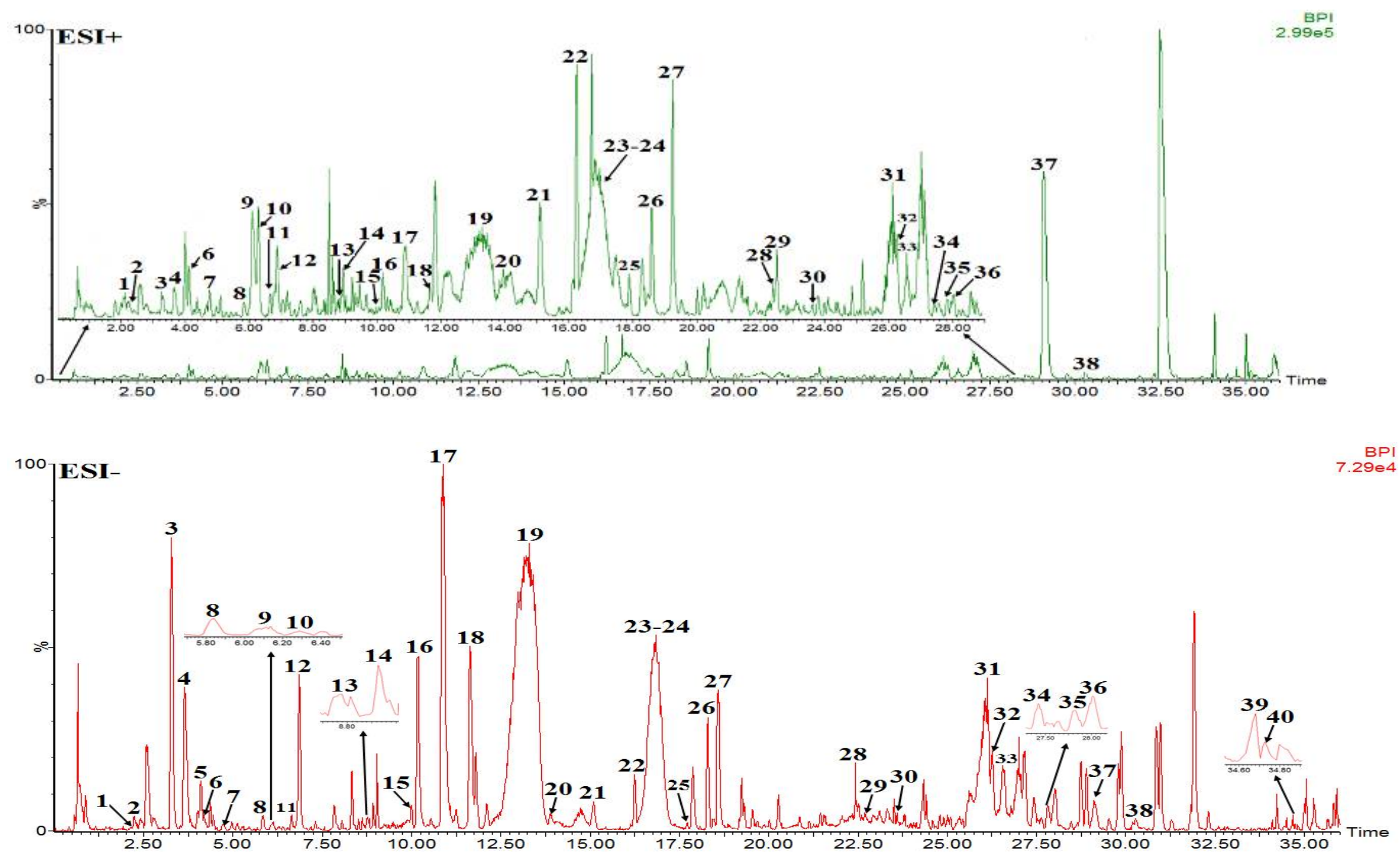

Figure 1: The base peak ion current chromatogram of $P$. hookeri in ESI+ and ESI- modes 


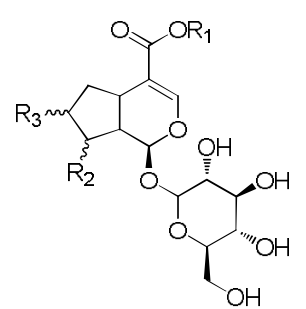

$1 \mathrm{R}_{1}=-\mathrm{H}_{2}, \mathrm{R}_{2}=-\alpha \mathrm{CH}_{3}, \mathrm{R}_{3}=-\beta \mathrm{OH}$

$3 \mathrm{R}_{1}=-\mathrm{II}, \mathrm{R}_{2}=-\beta \mathrm{CH} 3, \mathrm{R}_{3}=-\beta \mathrm{OH}$

$8 \mathrm{R}_{1}=-\mathrm{CH}_{3}, \mathrm{R}_{2}=-\beta \mathrm{CH}_{3}, \mathrm{R}_{3}=-\alpha \mathrm{OH}$

$10 \mathrm{R}_{1}=-\mathrm{CH}_{3}, \mathrm{R}_{2}=-\beta \mathrm{CH}_{3}, \mathrm{R}_{3}=-\beta \mathrm{OH}$<smiles>O=C(O)C=Cc1ccc(O)c(O)c1</smiles><smiles>C=CC1OC=C(C(=O)OC)C2CC(OC(=O)C3=COC(OC4OC(CO)C(O)C(O)C4O)C(CC=O)C3C)C(O)C12</smiles>

$19 \mathrm{R}_{1}=-\mathrm{Glc}$ $23 \mathrm{R}_{1}=-\mathrm{II}$<smiles>C=CC1C(OC2OC(CO)[C@@H](O)C(O)[C@H]2O)OC=C(C(=O)OC2CC3C(COC(O)C3C(=O)OC)C2C)C1CC=O</smiles>

21

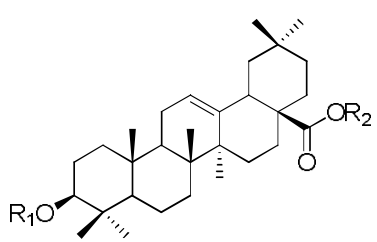

$28 \mathrm{R}_{1}=-\mathrm{Xyl}{ }^{2} \mathrm{Rha}^{{ }^{3} \mathrm{Xyl}}{ }^{4} \mathrm{Glc}^{4} \mathrm{Xyl}^{2} \mathrm{Rha}, \mathrm{R}_{2}=-\mathrm{Glc}^{6} \mathrm{Glc}$

$29 \mathrm{R}_{1}=-\mathrm{Xyl}{ }^{2} \mathrm{Rha}^{2} \mathrm{Xyl}^{4} \mathrm{Glc}^{4} \mathrm{Xyl}^{-} \mathrm{R}_{2}=-\mathrm{Glc}^{6} \mathrm{Glc}$

$30 \mathrm{R}_{1}=-\mathrm{Xyl}{ }^{2}{ }^{2} \mathrm{Rha}{ }^{3}{ }^{3} \mathrm{Xyl}{ }^{4} \mathrm{Glc}, \mathrm{R}_{2}=-\mathrm{Glc}{ }^{6} \mathrm{Glc}$

$31 \mathrm{R}_{1}=\mathrm{Xyl}{ }^{2} \mathrm{Rha}^{3} \mathrm{Xyl}{ }^{4} \mathrm{Glc}^{4}{ }^{4} \mathrm{Xy}{ }^{2}{ }^{2} \mathrm{Rha}, \mathrm{R}_{2}=-\mathrm{I}$

$32 \mathrm{R}_{1}=-\mathrm{Xyl}^{2}{ }^{2} \mathrm{Rha}^{3}{ }^{3} \mathrm{Xyl}^{4}{ }^{4} \mathrm{Glc}^{4}{ }^{4} \mathrm{Xyl}, \mathrm{R}_{2}=-\mathrm{H}$

$33 \mathrm{R}_{1}=-\mathrm{Xyl}{ }^{2} \mathrm{Rha}{ }^{3}{ }^{3} \mathrm{Xyl}{ }^{4} \mathrm{Glc}, \mathrm{R}_{2}=-\mathrm{H}$

$34 \mathrm{R}_{1}=\mathrm{Xyl}{ }^{\underline{3}} \mathrm{Rha}^{\underline{3}} \mathrm{Xyl}{ }^{\underline{3}} \mathrm{Rha}^{3} \mathrm{Xyl}, \mathrm{R}_{2}=-\mathrm{II}$ 2
$\mathrm{X} y \mathrm{l}$

$35 \mathrm{R}_{1}=-\mathrm{Xyl}{ }^{2}{ }^{\mathrm{Rha}}{ }^{3} \mathrm{Xyl}{ }^{4} \mathrm{Rha}, \mathrm{R}_{2}=-\mathrm{H}$

$36 \mathrm{R}_{1}=-\mathrm{Xyl}{ }^{2} \mathrm{Rha}^{\frac{3}{}} \mathrm{Xyl}, \mathrm{R}_{2}=-\mathrm{II}$

$37 \mathrm{R}_{1}=-\mathrm{Xyl}{ }^{2} \mathrm{Rha}, \mathrm{R}_{2}=-\mathrm{H}$

$38 \mathrm{R}_{1}=-\mathrm{Xyl}, \mathrm{R}_{2}=-\mathrm{H}$

$39 \mathrm{R}_{1}=-\mathrm{II}, \mathrm{R}_{2}=-\mathrm{II}$
$2 \mathrm{R}_{1}=$-Caffeoyl, $\mathrm{R}_{2}=-\mathrm{II}, \mathrm{R}_{3}=-\mathrm{II}$

$4 \mathrm{R}_{1}=-\mathrm{H}, \mathrm{R}_{2}=-\mathrm{H}, \mathrm{R}_{3}=$-Caffeoyl

$6 \mathrm{R}_{1}=-\mathrm{H}, \mathrm{R}_{2}=$-Caffeoyl, $\mathrm{R}_{3}=-\mathrm{H}$

$15 \mathrm{R}_{1}=$-II, $\mathrm{R}_{2}=$-Caffeoyl, $\mathrm{R}_{3}=$-Caffeoyl

$16 \mathrm{R}_{1}=$-Caffeoyl, $\mathrm{R}_{2}=-$ II, $\mathrm{R}_{3}=$-Caffeoyl

$18 \mathrm{R}_{1}=$-Caffeoyl, $\mathrm{R}_{2}=$-Caffeoyl, $\mathrm{R}_{3}=-\mathrm{H}$

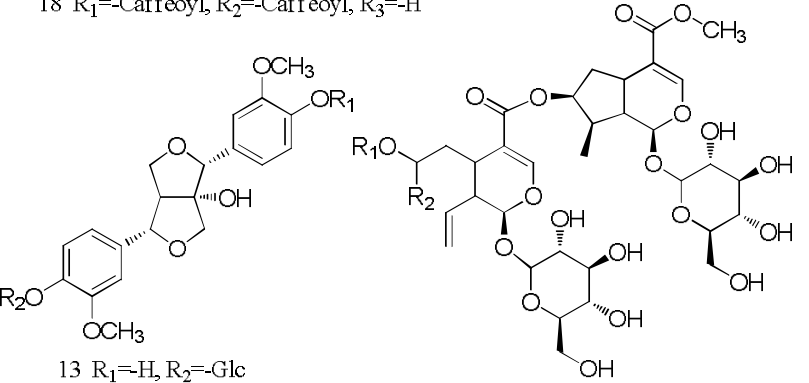

$14 \mathrm{R}_{1}=-\mathrm{Glc}, \mathrm{R}_{2}=-\mathrm{II}$

$17 \mathrm{R}_{1}=-\mathrm{H}, \mathrm{R}_{2}=-\mathrm{H}$

$25 \mathrm{R}_{1}=-\mathrm{CH}_{3}, \mathrm{R}_{2}=-\mathrm{OCH} I_{3}$

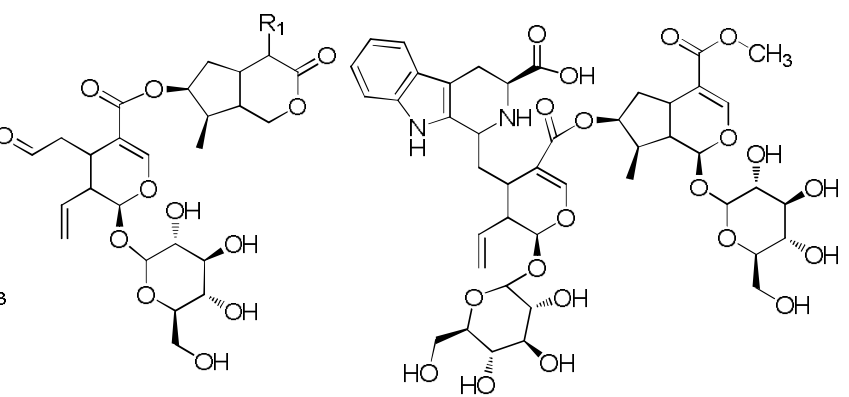

$20 \mathrm{R}_{1}=-\mathrm{H}$

$24 \mathrm{R}_{1}=-\mathrm{COOCH}_{3}$

22

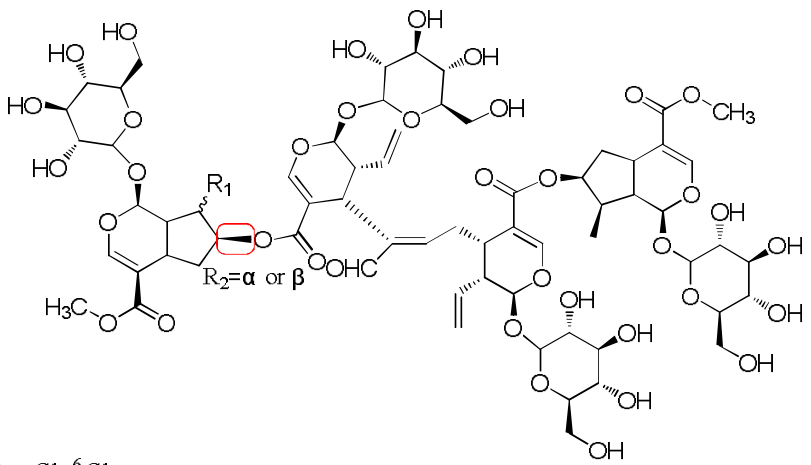

$26 \mathrm{R}_{1}=-\mathrm{aCH} 3, \mathrm{R}_{2}=-\mathrm{a}$

$27 \mathrm{R}_{1}=-\beta \mathrm{CH} 3, \mathrm{R}_{2}=-\beta$

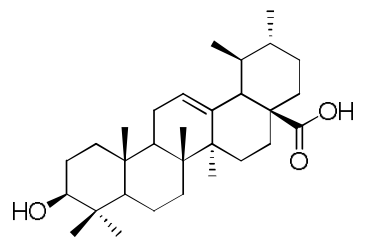

40

Figure 2: Chemical structures of compounds identified 
Table 1: ESI-TOF/MS data for compounds identified from $P$. hookeri

\begin{tabular}{|c|c|c|c|c|c|c|}
\hline No. & $\begin{array}{l}\mathrm{T}_{\mathrm{R}} \\
\text { (min) }\end{array}$ & Formula & $\begin{array}{l}\text { MS } \\
\text { (error in ppm) }\end{array}$ & Fragment ions & Identification & Ref. \\
\hline $1 \mathrm{~b}$ & 2.17 & $\mathrm{C}_{16} \mathrm{H}_{24} \mathrm{O}_{10}$ & $\begin{array}{l}375.1294[\mathrm{M}-\mathrm{H}]^{-} \\
(0.8)\end{array}$ & $397.1082[\mathrm{M}+\mathrm{Na}-2 \mathrm{H}]^{-}, 213.0768[\mathrm{M}-\mathrm{H}-\mathrm{Glc}]^{\prime}, 169.0846\left[\mathrm{M}-\mathrm{H}-\mathrm{Glc}-\mathrm{CO}_{2}\right]^{-}$ & 8-epi-loganin acid & [13] \\
\hline $2 b$ & 2.25 & $\mathrm{C}_{16} \mathrm{H}_{18} \mathrm{O}_{9}$ & $\begin{array}{l}353.0870[\mathrm{M}-\mathrm{H}]^{-} \\
(-0.8)\end{array}$ & $\begin{array}{l}173.0441\left[\mathrm{M}-\mathrm{H}-\mathrm{C}_{9} \mathrm{H}_{6} \mathrm{O}_{3}-\mathrm{H}_{2} \mathrm{O}\right]^{-}, 161.0235\left[\mathrm{C}_{9} \mathrm{H}_{8} \mathrm{O}_{4}-\mathrm{H}-\mathrm{H}_{2} \mathrm{O}\right] \\
135.0445\left[\mathrm{C}_{9} \mathrm{H}_{8} \mathrm{O}_{4}-\mathrm{H}_{-}-\mathrm{CO}_{2}\right]^{-}\end{array}$ & Neochlorogenic acid & [14] \\
\hline $3 a$ & 3.29 & $\mathrm{C}_{16} \mathrm{H}_{24} \mathrm{O}_{10}$ & $\begin{array}{l}375.1298[\mathrm{M}-\mathrm{H}]^{-} \\
(-2.7)\end{array}$ & $\begin{array}{l}773.2469[2 \mathrm{M}+\mathrm{Na}-2 \mathrm{H}]^{-}, 751.2656[2 \mathrm{M}-\mathrm{H}]^{-}, 213.0759[\mathrm{M}-\mathrm{H}-\mathrm{Glc}]^{-}, 169.0859 \\
{\left[\mathrm{M}-\mathrm{H}-\mathrm{Glc}-\mathrm{CO}_{2}\right]^{-}, 151.0752\left[\mathrm{M}-\mathrm{H}-\mathrm{Glc}-\mathrm{CO}_{2}-\mathrm{H}_{2} \mathrm{O}\right]^{-}, 113.0239\left[\mathrm{M}-\mathrm{H}-\mathrm{Glc}^{-} \mathrm{CO}_{2^{-}}\right.} \\
\mathrm{C}_{3} \mathrm{H}_{4} \mathrm{O}^{-}\end{array}$ & Loganic acid & [13] \\
\hline $4 a$ & 3.64 & $\mathrm{C}_{16} \mathrm{H}_{18} \mathrm{O}_{9}$ & $\begin{array}{l}353.0866[\mathrm{M}-\mathrm{H}]^{-} \\
(-2.0)\end{array}$ & $\begin{array}{l}729.1613[2 \mathrm{M}+\mathrm{Na}-2 \mathrm{H}]^{-}, 707.1810[2 \mathrm{M}-\mathrm{H}]^{-}, 375.0866[\mathrm{M}+\mathrm{Na}-2 \mathrm{H}]^{-}, 191.0560 \\
{\left[\mathrm{M}-\mathrm{H}-\mathrm{C}_{9} \mathrm{H}_{6} \mathrm{O}_{3}\right]^{-}, 179.0350\left[\mathrm{C}_{9} \mathrm{H}_{8} \mathrm{O}_{4}-\mathrm{H}\right]^{-}}\end{array}$ & Chlorogenic acid & [14] \\
\hline $5 b$ & 4.10 & $\mathrm{C}_{9} \mathrm{H}_{8} \mathrm{O}_{4}$ & $\begin{array}{l}179.0351[\mathrm{M}-\mathrm{H}]^{-} \\
(3.9)\end{array}$ & $201.0152[\mathrm{M}+\mathrm{Na}-2 \mathrm{H}], 135.0452\left[\mathrm{M}-\mathrm{H}-\mathrm{CO}_{2}\right]^{-}$ & Caffeic acid & [13] \\
\hline $6 b$ & 4.14 & $\mathrm{C}_{16} \mathrm{H}_{18} \mathrm{O}_{9}$ & $\begin{array}{l}353.0866[\mathrm{M}-\mathrm{H}]^{-} \\
(-2.0)\end{array}$ & $\begin{array}{l}375.0877[\mathrm{M}+\mathrm{Na}-2 \mathrm{H}]^{-}, 191.0556\left[\mathrm{M}-\mathrm{H}-\mathrm{C}_{9} \mathrm{H}_{6} \mathrm{O}_{3}\right]^{-}, 179.0345\left[\mathrm{C}_{9} \mathrm{H}_{8} \mathrm{O}_{4}-\mathrm{H}\right]^{-}, \\
173.0452\left[\mathrm{M}-\mathrm{H}-\mathrm{C}_{9} \mathrm{H}_{6} \mathrm{O}_{3}-\mathrm{H}_{2} \mathrm{O}\right]^{-}\end{array}$ & Cryptochlorogenic acid & [14] \\
\hline $7 \mathrm{~b}$ & 4.74 & $\mathrm{C}_{16} \mathrm{H}_{22} \mathrm{O}_{10}$ & $\begin{array}{l}373.1124[\mathrm{M}-\mathrm{H}]^{-} \\
(-2.9)\end{array}$ & $\begin{array}{l}769.2156[2 \mathrm{M}+\mathrm{Na}-2 \mathrm{H}]^{-}, 747.2343[2 \mathrm{M}-\mathrm{H}]^{-}, 211.0236[\mathrm{M}-\mathrm{H}-\mathrm{Glc}]^{-} \\
\text {167.0701 [M-H-Glc-CO }\end{array}$ & Swertimarin & [13] \\
\hline $8 b$ & 5.84 & $\mathrm{C}_{17} \mathrm{H}_{26} \mathrm{O}_{10}$ & $\begin{array}{l}389.1462[\mathrm{M}-\mathrm{H}]^{-} \\
(3.6)\end{array}$ & $227.0904[\mathrm{M}-\mathrm{H}-\mathrm{Glc}]^{-}$ & 7-epi-loganin & [13] \\
\hline $9 a$ & 6.10 & $\mathrm{C}_{16} \mathrm{H}_{22} \mathrm{O}_{9}$ & $\begin{array}{l}357.1201[\mathrm{M}-\mathrm{H}]^{-} \\
(4.2)\end{array}$ & $\begin{array}{l}715.2451[2 \mathrm{M}-\mathrm{H}]^{-}, 403.1239[\mathrm{M}-\mathrm{H}+\mathrm{HCOOH}]^{-}, 297.1115\left[\mathrm{M}-\mathrm{H}_{-} \mathrm{C}_{2} \mathrm{H}_{4} \mathrm{O}_{2}\right]^{-} \\
195.0660[\mathrm{M}-\mathrm{H}-\mathrm{Glc}]^{-}, 125.0242\left[\mathrm{M}-\mathrm{H}-\mathrm{Glc}_{4} \mathrm{H}_{6} \mathrm{O}\right]^{-}\end{array}$ & Sweroside & [15] \\
\hline $10 a$ & 6.29 & $\mathrm{C}_{17} \mathrm{H}_{26} \mathrm{O}_{10}$ & $\begin{array}{l}779.2971[2 \mathrm{M}-\mathrm{H}]^{-} \\
(-0.4)\end{array}$ & $\begin{array}{l}801.1882[2 \mathrm{M}+\mathrm{Na}-2 \mathrm{H}]^{-}, 435.1503[\mathrm{M}-\mathrm{H}+\mathrm{HCOOH}]^{-}, 227.0926[\mathrm{M}-\mathrm{H}-\mathrm{Glc}]^{-}, \\
209.0838\left[\mathrm{M}+\mathrm{H}-\mathrm{Glc}-\mathrm{H}_{2} \mathrm{O}\right]^{-}\end{array}$ & Loganin & [13] \\
\hline $11 b$ & 6.65 & $\mathrm{C}_{22} \mathrm{H}_{32} \mathrm{O}_{14}$ & $\begin{array}{l}519.1710[\mathrm{M}-\mathrm{H}]^{-} \\
(-0.8)\end{array}$ & 1039.3473 [2M-H], 357.1199 [M-H-Glc], 195.0662 [M-H-Glc-Glc] & Dipsanosides H & [16] \\
\hline $12 \mathrm{ab}$ & 6.85 & $\mathrm{C}_{21} \mathrm{H}_{30} \mathrm{O}_{13}$ & $\begin{array}{l}489.1609[\mathrm{M}-\mathrm{H}]^{-} \\
(0.2)\end{array}$ & 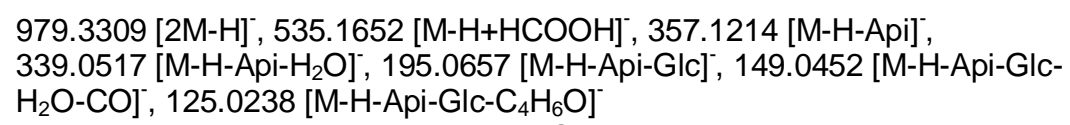 & 6'-apiofuranosylsweroside & {$[16]$} \\
\hline 13 & 8.80 & $\mathrm{C}_{26} \mathrm{H}_{32} \mathrm{O}_{12}$ & $\begin{array}{l}535.1815[\mathrm{M}-\mathrm{H}]^{-} \\
(-0.2)\end{array}$ & $557.1970[\mathrm{M}+\mathrm{Na}-2 \mathrm{H}]^{-}, 373.1283[\mathrm{M}-\mathrm{H}-\mathrm{GlC}]^{-}$ & 8-hydroxylpinoresionl-4'-glucoside & {$[10]$} \\
\hline 14 & 8.94 & $\mathrm{C}_{26} \mathrm{H}_{32} \mathrm{O}_{12}$ & $\begin{array}{l}535.1865[\mathrm{M}-\mathrm{H}]^{-} \\
(5.0)\end{array}$ & $557.2025[\mathrm{M}+\mathrm{Na}-2 \mathrm{H}]^{-}, 373.1291[\mathrm{M}-\mathrm{H}-\mathrm{Glc}]^{-}$ & 8'-hydroxylpinoresionl-4-glucoside & [10] \\
\hline $15 a b$ & 10.01 & $\mathrm{C}_{25} \mathrm{H}_{24} \mathrm{O}_{12}$ & $\begin{array}{l}515.1208[\mathrm{M}-\mathrm{H}]^{-} \\
(3.5)\end{array}$ & $\begin{array}{l}\text { 1053.2207 [2M+Na-2H] }, 537.1027[\mathrm{M}+\mathrm{Na}-2 \mathrm{H}]^{-}, 353.0879\left[\mathrm{M}-\mathrm{H}-\mathrm{C}_{9} \mathrm{H}_{6} \mathrm{O}_{3}\right]^{-} \\
\text {191.0549 }\left[\mathrm{M}-\mathrm{H}-2 \mathrm{C}_{9} \mathrm{H}_{6} \mathrm{O}_{3}\right]^{-}\end{array}$ & 3,4-dicaffeoylquinic acid & [14] \\
\hline $16 a b$ & 10.19 & $\mathrm{C}_{25} \mathrm{H}_{24} \mathrm{O}_{12}$ & $\begin{array}{l}515.1183[\mathrm{M}-\mathrm{H}]^{-} \\
(-1.4)\end{array}$ & $1031.2461[2 \mathrm{M}-\mathrm{H}]^{-}, 353.0869\left[\mathrm{M}-\mathrm{H}-\mathrm{C}_{9} \mathrm{H}_{6} \mathrm{O}_{3}\right]^{-}, 191.0557\left[\mathrm{M}-\mathrm{H}-2 \mathrm{C}_{9} \mathrm{H}_{6} \mathrm{O}_{3}\right]^{-}$ & 3,5-dicaffeoylquinic acid & [14] \\
\hline $17 a$ & 10.89 & $\mathrm{C}_{33} \mathrm{H}_{48} \mathrm{O}_{19}$ & $\begin{array}{l}747.2701[\mathrm{M}-\mathrm{H}]^{-} \\
(-1.5)\end{array}$ & $1495.5585[2 \mathrm{M}-\mathrm{H}]^{-}, 585.2175[\mathrm{M}-\mathrm{H}-\mathrm{Glc}]^{-}, 423.1235\left[[\mathrm{M}-\mathrm{H}-\mathrm{Glc}-\mathrm{Glc}]^{-}\right.$ & Sylvestroside I & {$[10]$} \\
\hline $18 a b$ & 11.70 & $\mathrm{C}_{25} \mathrm{H}_{24} \mathrm{O}_{12}$ & $\begin{array}{l}515.1197[\mathrm{M}-\mathrm{H}]^{-} \\
(1.4)\end{array}$ & $\begin{array}{l}\text { 1053.2296 [2M+Na-2H] }, 1031.2493[2 \mathrm{M}-\mathrm{H}]^{-}, 537.1018[\mathrm{M}+\mathrm{Na}-2 \mathrm{H}]^{-}, \\
353.0872\left[\mathrm{M}-\mathrm{H}-\mathrm{C}_{9} \mathrm{H}_{6} \mathrm{O}_{3}\right]^{-}, 191.0549\left[\mathrm{M}-\mathrm{H}-2 \mathrm{C}_{9} \mathrm{H}_{6} \mathrm{O}_{3}\right]^{-}\end{array}$ & 4,5-dicaffeoylquinic acid & {$[14]$} \\
\hline
\end{tabular}


Table 1: ESI-TOF/MS data for compounds identified from P. hookeri (contd)

\begin{tabular}{|c|c|c|c|c|c|c|}
\hline No. & $\begin{array}{l}\mathrm{T}_{\mathrm{R}} \\
\text { (min) }\end{array}$ & Formula & $\begin{array}{l}\text { MS } \\
\text { (error in ppm) }\end{array}$ & Fragment ions & Identification & Ref. \\
\hline $19 a$ & 13.34 & $\mathrm{C}_{33} \mathrm{H}_{46} \mathrm{O}_{19}$ & $\begin{array}{l}745.2576[\mathrm{M}-\mathrm{H}] \\
(2.8)\end{array}$ & $\begin{array}{l}1491.5344 \text { [2M-H]', } 583.2040[\mathrm{M}-\mathrm{H}-\mathrm{Glc}]^{-} \\
421.1163 \text { [M-H-Glc-Glc] }\end{array}$ & Cantleyoside & {$[11]$} \\
\hline 20 & 13.90 & $\mathrm{C}_{25} \mathrm{H}_{34} \mathrm{O}_{12}$ & $\begin{array}{l}525.1973[\mathrm{M}-\mathrm{H}]^{-} \\
(0.2)\end{array}$ & $\begin{array}{l}1051.4105[2 \mathrm{M}-\mathrm{H}]^{-}, 571.2026[\mathrm{M}-\mathrm{H}+\mathrm{HCOOH}]^{-} \\
363.1512[\mathrm{M}-\mathrm{H}-\mathrm{GlC}]^{-}\end{array}$ & Laciniatoside II & [17] \\
\hline 21 & 15.10 & $\mathrm{C}_{27} \mathrm{H}_{38} \mathrm{O}_{14}$ & $\begin{array}{l}585.2172[\mathrm{M}-\mathrm{H}]^{-} \\
(-1.9)\end{array}$ & $631.2221[\mathrm{M}-\mathrm{H}+\mathrm{HCOOH}]^{-}, 423.1652[\mathrm{M}-\mathrm{H}-\mathrm{Glc}]^{-}$ & Laciniatoside I & [17] \\
\hline $22 b$ & 16.24 & $\mathrm{C}_{44} \mathrm{H}_{56} \mathrm{~N}_{2} \mathrm{O}_{20}$ & $\begin{array}{l}933.3507[\mathrm{M}+\mathrm{H}]^{+} \\
(0.2)\end{array}$ & $771.2957[\mathrm{M}+\mathrm{H}-\mathrm{Glc}]^{+}, 609.2066[\mathrm{M}+\mathrm{H}-\mathrm{Glc}-\mathrm{Glc}]^{+}$ & Pterocephaline & [18] \\
\hline 23 & 16.71 & $\mathrm{C}_{27} \mathrm{H}_{36} \mathrm{O}_{14}$ & $\begin{array}{l}583.2030[\mathrm{M}-\mathrm{H}]^{-} \\
(0.5)\end{array}$ & $1189.3997[2 \mathrm{M}+\mathrm{Na}-2 \mathrm{H}]^{-}, 421.1485[\mathrm{M}-\mathrm{H}-\mathrm{Glc}]^{-}$ & Sylvestroside III & {$[17]$} \\
\hline 24 & 16.84 & $\mathrm{C}_{27} \mathrm{H}_{36} \mathrm{O}_{14}$ & $\begin{array}{l}583.2030[\mathrm{M}-\mathrm{H}]^{-} \\
(0.5)\end{array}$ & $1189.3986[2 \mathrm{M}+\mathrm{Na}-2 \mathrm{H}]^{-}, 421.1462[\mathrm{M}-\mathrm{H}-\mathrm{Glc}]^{-}$ & Sylvestroside VI & [17] \\
\hline $25 a$ & 17.72 & $\mathrm{C}_{35} \mathrm{H}_{52} \mathrm{O}_{20}$ & $\begin{array}{l}791.2967[\mathrm{M}-\mathrm{H}]^{-} \\
(-0.9)\end{array}$ & $629.2448[\mathrm{M}-\mathrm{H}-\mathrm{Glc}]^{-}$ & Triplostoside A & {$[14]$} \\
\hline $26 a$ & 18.28 & $\mathrm{C}_{66} \mathrm{H}_{90} \mathrm{O}_{37}$ & $\begin{array}{l}1473.5153[\mathrm{M}-\mathrm{H}]^{-} \\
(4.8)\end{array}$ & $\begin{array}{l}\left.\left.1311.4646[\mathrm{M}-\mathrm{H}-\mathrm{Glc}]^{-}, 1131.3984 \text { [M-H-2Glc-H}_{2} \mathrm{O}\right]^{-}, 969.2977 \text { [M-H-3Glc- } \mathrm{H}_{2} \mathrm{O}\right]^{-} \\
825.2862 \text { [M-H-4Glc] }^{-}\end{array}$ & Dipsanosides B & [14] \\
\hline $27 a$ & 18.58 & $\mathrm{C}_{66} \mathrm{H}_{90} \mathrm{O}_{37}$ & $\begin{array}{l}1473.5129[\mathrm{M}-\mathrm{H}]^{-} \\
(3.1)\end{array}$ & $\begin{array}{l}\left.\text { 1311.4545 [M-H-Glc] }]^{-}, 1149.3488 \text { [M-H-2Glc]', } 969.2973 \text { [M-H-3Glc - } \mathrm{H}_{2} \mathrm{O}\right]^{-} \\
807.2715\left[\mathrm{M}-\mathrm{H}-4 \mathrm{Glc}-\mathrm{H}_{2} \mathrm{O}\right]^{-}\end{array}$ & Dipsanosides A & [14] \\
\hline 28 & 22.44 & $\mathrm{C}_{75} \mathrm{H}_{122} \mathrm{O}_{38}$ & $\begin{array}{l}1629.7534[\mathrm{M}-\mathrm{H}]^{-} \\
(-0.1)\end{array}$ & $\begin{array}{l}1305.6500 \text { [M-H-2Glc] , } 1159.6040 \text { [M-H-2Glc-Rha], } 1027.5486 \text { [M-H-2Glc-Rha- } \\
\text { Xyl], } 865.4760 \text { [M-H-3Glc-Rha-Xyl]", 733.4683 [M-H-3Glc-Rha-2Xyl]", 587.3968 } \\
\text { [M-H-3Glc-2Rha-2Xyl]", 455.3522 [M-H-3Glc-2Rha-3Xyl] }\end{array}$ & Hookerosides C & [19] \\
\hline 29 & 22.50 & $\mathrm{C}_{69} \mathrm{H}_{112} \mathrm{O}_{34}$ & $\begin{array}{l}1483.7013[\mathrm{M}-\mathrm{H}]^{-} \\
(3.8)\end{array}$ & $\begin{array}{l}1159.5927 \text { [M-H-2Glc]', } 1027.5481 \text { [M-H-2Glc -Xyl], 865.4907 [M-H-3Glc-Xyl]', } \\
\text { 733.4633 [M-H-3Glc-2Xyl], 587.3981 [M-H-3Glc-Rha-2Xyl], } 455.3550 \text { [M-H-3Glc- } \\
\text { Rha-3Xyl] }\end{array}$ & Hookerosides B & [19] \\
\hline 30 & 23.60 & $\mathrm{C}_{64} \mathrm{H}_{104} \mathrm{O}_{30}$ & $\begin{array}{l}1351.6509[\mathrm{M}-\mathrm{H}]^{-} \\
(-1.8)\end{array}$ & $\begin{array}{l}1027.5470 \text { [M-H-2Glc] }{ }^{-}, 865.4976 \text { [M-H-3Glc]-, 733.4747 [M-H-3Glc-Xyl]', } \\
587.3919 \text { [M-H-3Glc-Xyl-Rha]", 455.3521 [M-H-3Glc-2Xyl-Rha] }\end{array}$ & Hookerosides A & [19] \\
\hline 31 & 26.07 & $\mathrm{C}_{63} \mathrm{H}_{102} \mathrm{O}_{28}$ & $\begin{array}{l}1305.6544[\mathrm{M}-\mathrm{H}]^{-} \\
(5.0)\end{array}$ & 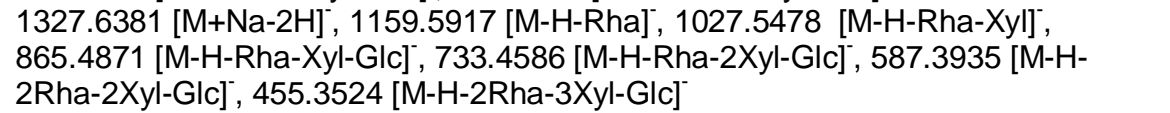 & Hookerosides D & [19] \\
\hline 32 & 26.25 & $\mathrm{C}_{57} \mathrm{H}_{92} \mathrm{O}_{24}$ & $\begin{array}{l}1159.5923[\mathrm{M}-\mathrm{H}]^{-} \\
(2.0)\end{array}$ & $\begin{array}{l}1181.5713 \text { [M+Na-2H], } 1027.5490 \text { [M-H-Xyl], } 865.4960 \text { [M-H-Xyl-Glc]-, } 733.4566 \\
\text { [M-H-2Xyl-Glc] }^{-}, 587.4064 \text { [M-H-Rha-2Xyl-Glc]', } 455.3505 \text { [M-H-Rha-3Xyl-Glc] }\end{array}$ & $\begin{array}{l}\text { Oleanolic acid } 3-X y l(1 \rightarrow 4)- \\
\text { Glc }(1 \rightarrow 4)-X y l(1 \rightarrow 3)-R h a(1 \rightarrow 2)-X y l\end{array}$ & [19] \\
\hline 33 & 26.55 & $\mathrm{C}_{52} \mathrm{H}_{84} \mathrm{O}_{20}$ & $\begin{array}{l}1027.5485[\mathrm{M}-\mathrm{H}]^{-} \\
(0.7)\end{array}$ & 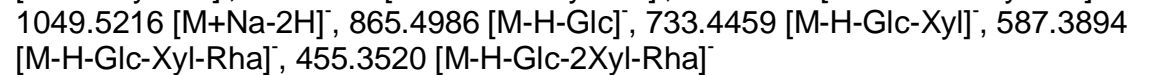 & $\begin{array}{l}\text { Oleanolic acid 3-Glc }(1 \rightarrow 4)- \\
\text { Xyl }(1 \rightarrow 3)-R h a(1 \rightarrow 2)-X y l\end{array}$ & [19] \\
\hline 34 & 27.43 & $\mathrm{C} 62 \mathrm{H} 100 \mathrm{O} 27$ & $\begin{array}{l}1275.6399[\mathrm{M}-\mathrm{H}]- \\
(2.0)\end{array}$ & $\begin{array}{l}1297.6207 \text { [M+Na-2H]-, } 1143.5941 \text { [M-H-Xyl]-, } 997.5147 \text { [M-H-Xyl-Rha]-, } \\
865.5060 \text { [M-H-2Xyl-Rha]-, } 719.4508 \text { [M-H-2Xyl-2Rha]-, 587.3959 [M-H-3Xyl- } \\
\text { 2Rha]-, } 455.3520 \text { [M-H-4Xyl-2Rha]- }\end{array}$ & Rivularicin & [20] \\
\hline $35 b$ & 27.82 & C52H84O19 & $\begin{array}{l}1011.5532[\mathrm{M}-\mathrm{H}]- \\
(0.3)\end{array}$ & $\begin{array}{l}\text { 1033.5337 [M+Na-2H]-, } 865.4962 \text { [M-H-Rha]-, } 733.4523 \text { [M-H-Rha-Xyl]-, } 587.3956 \\
\text { [M-H-2Rha-Xyl]-, 455.3532 [M-H-2Rha-2Xyl]- }\end{array}$ & Triploside G & [21] \\
\hline
\end{tabular}


Table 1: ESI-TOF/MS data for compounds identified from P. hookeri (contd)

\begin{tabular}{|c|c|c|c|c|c|c|}
\hline No. & $\begin{array}{l}T_{R} \\
(\min ) \\
\end{array}$ & Formla & $\begin{array}{l}\text { MS } \\
\text { (error in ppm) }\end{array}$ & Fragment ions & Identification & Ref. \\
\hline 36 & 28.00 & $\mathrm{C}_{46} \mathrm{H}_{74} \mathrm{O}_{15}$ & $\begin{array}{l}865.4957[\mathrm{M}-\mathrm{H}]^{-} \\
(0.9)\end{array}$ & $\begin{array}{l}887.4765[\mathrm{M}+\mathrm{Na}-2 \mathrm{H}]^{-}, 733.4520[\mathrm{M}-\mathrm{H}-\mathrm{Xyl}]^{-}, 587.3954 \text { [M-H-Rha-Xyl]', } \\
455.3538\left[\mathrm{M}-\mathrm{H}-\mathrm{Rha}-2 \mathrm{Xyl}^{-}\right.\end{array}$ & $\begin{array}{l}\text { Oleanolic acid 3-Xyl }(1 \rightarrow 3)-\text { Rha( }(1 \rightarrow 2)- \\
\text { Xyl }\end{array}$ & [19] \\
\hline 37 & 29.15 & $\mathrm{C}_{41} \mathrm{H}_{66} \mathrm{O}_{11}$ & $733.4531[\mathrm{M}-\mathrm{H}]^{-}(0.5)$ & 587.3969 [M-H-Rha] $]^{-}, 455.3524$ [M-H-Rha-Xyl] & giganteaside D & [19] \\
\hline 38 & 30.33 & $\mathrm{C}_{35} \mathrm{H}_{56} \mathrm{O}_{7}$ & $587.3927[\mathrm{M}-\mathrm{H}]^{-}(-3.6)$ & $455.3567[\mathrm{M}-\mathrm{H}-\mathrm{Xyl}]^{-}$ & songoroside $A$ & [19] \\
\hline $39 a$ & 34.68 & $\mathrm{C}_{30} \mathrm{H}_{48} \mathrm{O}_{3}$ & $455.3539[\mathrm{M}-\mathrm{H}]^{-}(3.1)$ & & Oleanolic acid & [22] \\
\hline $40 \mathrm{a}$ & 34.73 & $\mathrm{C}_{30} \mathrm{H}_{48} \mathrm{O}_{3}$ & $455.3510[\mathrm{M}-\mathrm{H}]^{-}(-3.3)$ & & Ursolic acid & [22] \\
\hline
\end{tabular}




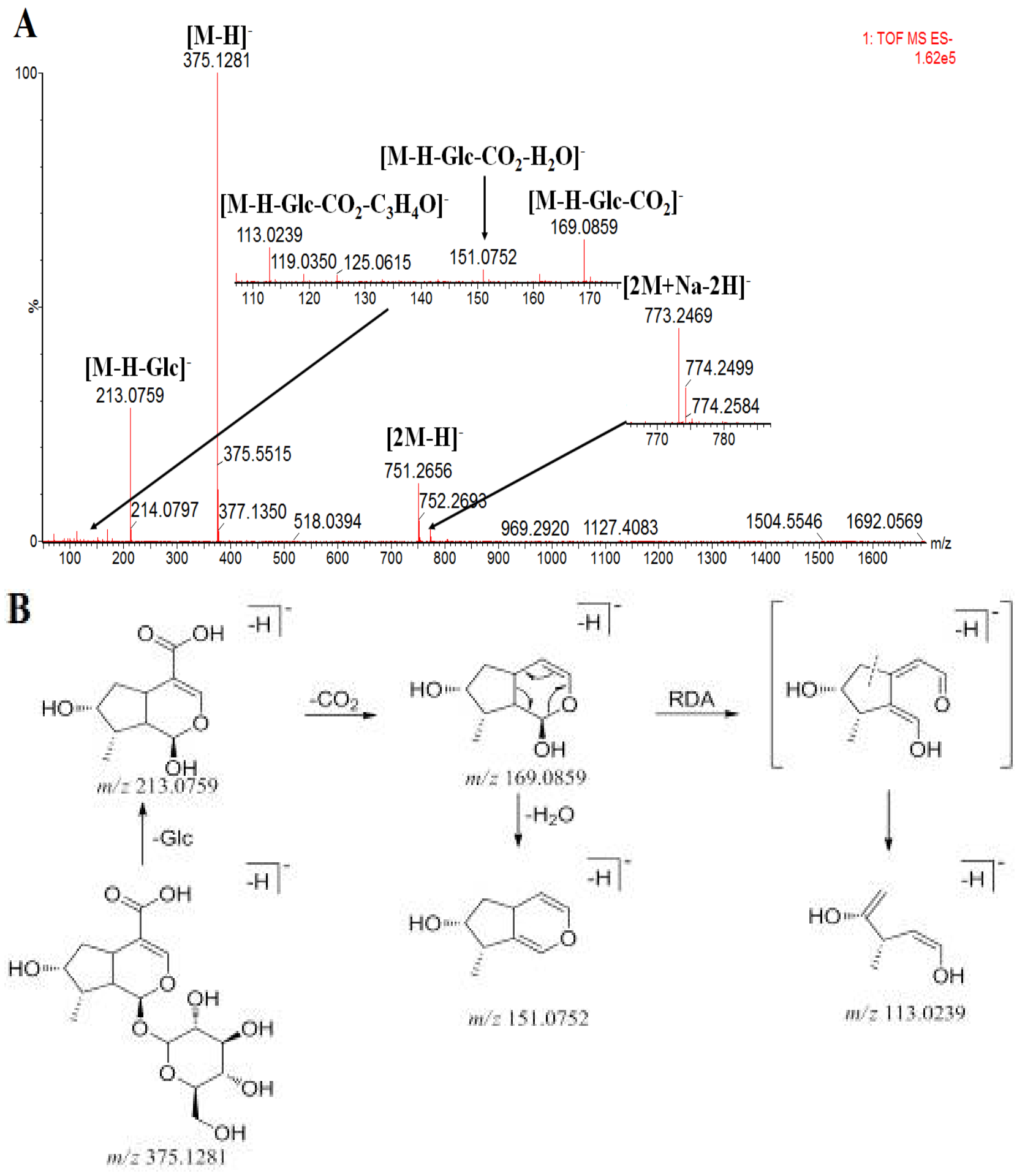

Figure 3: MS diagram (ESI-) and ions fragmentation pathways of loganic acid (3) 
A [M-H-caffeoyl]

353.0869
1: TOF MS ES-

$9.90 \mathrm{e} 4$

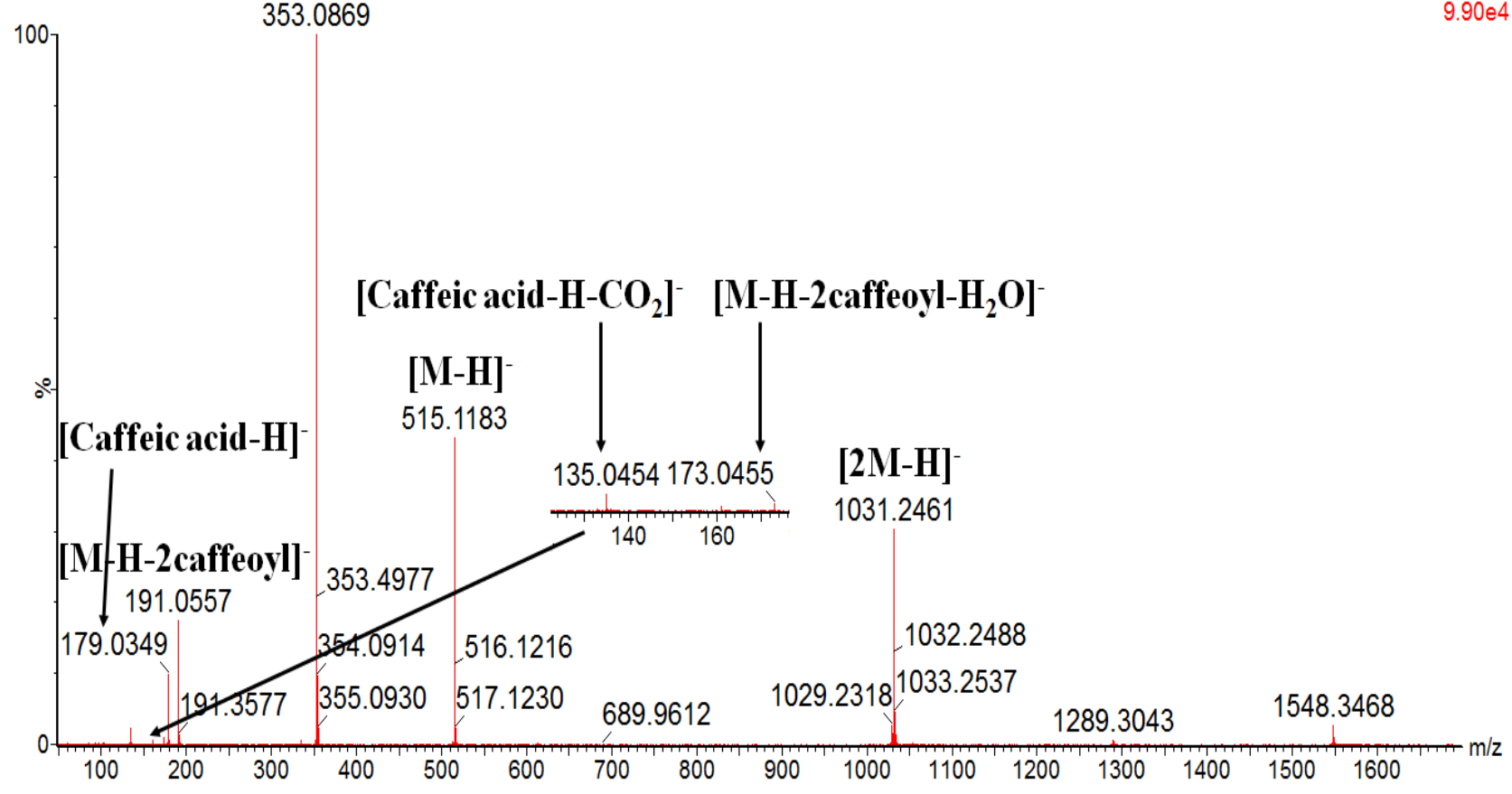

B<smiles>C=Cc1ccc(O)c(O)c1</smiles>

Figure 4: MS diagram (ESI-) and ions fragmentation pathways of 3,5-dicaffeoylquinic acid (16) 
A

$$
{ }^{100}
$$

100
A

113

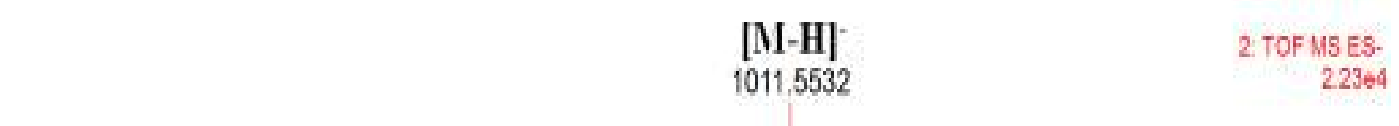

B

. 可

$-{ }^{*}{ }^{*}$

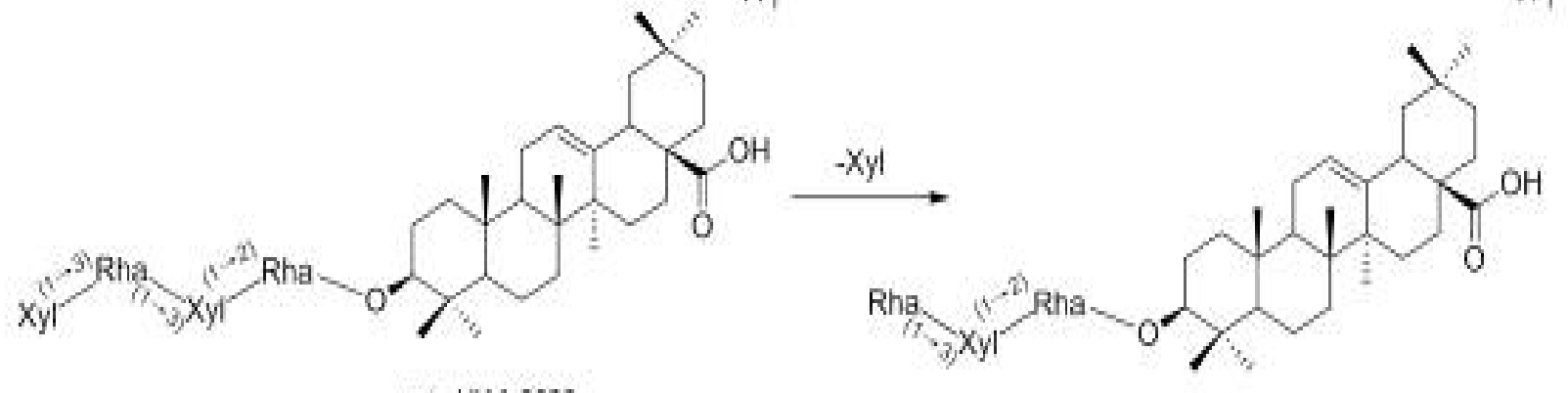

$m / 21011.5532$

$m / z 865,4962$

(Rha

$m / z 455,3532$

$m / 2587.3956$

$m / 2733.4523$

Figure 5: MS diagram (ESI-) and ions fragmentation pathways of triploside G (35) 
Table 2: Information on potential targets from compounds of $P$. hookeri

\begin{tabular}{|c|c|c|c|}
\hline No. & Gene & Protein & Compound \\
\hline 1 & CA2 & Carbonic anhydrase 2 & $\begin{array}{l}3,4,5,6,8,9,10,11,12,14,15,17 \\
18,19,20,21,22,23,25,27,30,32 \\
36,37,38,39,40\end{array}$ \\
\hline 2 & MAPK10 & Mitogen-activated protein kinase 10 & $\begin{array}{l}2,4,15,17,18,19,20,21,22,23,24 \\
26,27,28,32,33,37,38,40\end{array}$ \\
\hline 3 & PNP & Purine nucleoside phosphorylase & $\begin{array}{l}6,7,8,9,10,12,13,16,17,19,21,22 \\
23,24,26,27,28,29,30\end{array}$ \\
\hline 4 & BMP2 & Bone morphogenetic protein 2 & $\begin{array}{l}28,29,30,31,32,33,34,35,36,37 \\
38,39,40\end{array}$ \\
\hline 5 & GSTP1 & Glutathione S-transferase P & $\begin{array}{l}3,7,8,9,10,11,12,21,23,26,28,29 \\
30,35\end{array}$ \\
\hline 6 & ITGAL & Integrin alpha-L & $29,30,32,33,34,35,36,37,38,39$ \\
\hline 7 & AKR1C2 & Aldo-keto reductase family 1 member C2 & $31,32,33,34,35,36,37,38,39,40$ \\
\hline 8 & MAPK8 & Mitogen-activated protein kinase 8 & $2,15,17,18,19,20,21,22,23$ \\
\hline 9 & CASP3 & Caspase-3 & $1,3,9,11,12,36,37,38$ \\
\hline 10 & KDR & Vascular endothelial growth factor receptor 2 & $2,4,18,33,35,36,37,40$ \\
\hline 11 & BACE1 & Beta-secretase 1 & $2,4,5,15,18,38,39,40$ \\
\hline 12 & MTAP & S-methyl-5'-thioadenosine phosphorylase & $7,9,11,13,14,16$ \\
\hline 13 & CFB & Complement factor B & $15,17,20,22,24,27$ \\
\hline 14 & SELP & P-selectin & $28,29,30,31,32,33$ \\
\hline 15 & PIM1 & Serine/threonine-protein kinase pim-1 & $5,23,38,40$ \\
\hline 16 & MAPK1 & Mitogen-activated protein kinase 1 & $1,3,9,12,20$ \\
\hline 17 & CA1 & Carbonic anhydrase 1 & $12,13,18,31$ \\
\hline 18 & EGFR & Epidermal growth factor receptor & $1,5,6,14$ \\
\hline 19 & AMY1A & Alpha-amylase 1 & $2,7,16,18$ \\
\hline 20 & PDE4B & cAMP-specific 3',5'-cyclic phosphordiesterase 4B & $10,11,14$ \\
\hline 21 & NUDT9 & ADP-ribose pyrophosphatase, mitochondrial & 7,32 \\
\hline 22 & CHEK1 & Serine/threonine-protein kinase Chk1 & 13,14 \\
\hline 23 & MAPK14 & Mitogen-activated protein kinase 14 & 4,22 \\
\hline 24 & TGFBR2 & TGF-beta receptor type-2 & 4,34 \\
\hline 25 & $\mathrm{AR}$ & Androgen receptor & 15,39 \\
\hline 26 & MAPKAPK2 & MAP kinase-activated protein kinase 2 & 19,20 \\
\hline 27 & CDK2 & Cyclin-dependent kinase 2 & 5,12 \\
\hline 28 & CSNK2A1 & Casein kinase II subunit alpha & 3,8 \\
\hline 29 & F2 & Prothrombin & 2,32 \\
\hline 30 & CDK5R1 & Cyclin-dependent kinase 5 activator 1 & 6,16 \\
\hline 31 & PDE5A & cGMP-specific 3',5'-cyclic phosphodiesterase & 4 \\
\hline 32 & ICAM2 & Intercellular adhesion molecule 2 & 7 \\
\hline 33 & TGFBR1 & TGF-beta receptor type-1 & 10 \\
\hline 34 & CFD & Complement factor $\mathrm{D}$ & 10 \\
\hline 35 & CA12 & Carbonic anhydrase 12 & 11 \\
\hline 36 & PPARG & Peroxisome proliferator-activated receptor gamma & 24 \\
\hline 37 & CASP7 & Caspase-7 & 39 \\
\hline 38 & SRC & Proto-oncogene tyrosine-protein kinase Src & 1 \\
\hline
\end{tabular}

and pathways (Figure 6). Though the network diagram, a preliminary mechanism of the anti-RA of $P$. hookeri was obtained.

Table 3 show that the MAPK signaling pathway was involved in the largest number of targets. This pathway affects the release of inflammatory cytokines, and inhibits abnormal synaptic proliferation, thereby inhibition of RA bone erosion [25]. In addition, some cancer-related pathways were found, including those related to colorectal, prostate, and pancreatic cancers. This is consistent with a previous report [24].

\section{DISCUSSION}

The technique of UPLC-Q-TOF/MS has the preponderances of fast analysis, low detection limit and strong qualitative ability. It has been widely used in the analysis of the chemical composition of medicinal plants, and has become an important means of identifying various compounds. In the MS data analysis process, it is extremely important to identify the correct quasi-molecular ion. This is related to the accuracy of the results. In the $\mathrm{ESI}+$ mode, the quasi-molecular ion $[\mathrm{M}+\mathrm{Na}]^{+}$is easily formed, while the ESI- mode mainly forms the $[\mathrm{M}-\mathrm{H}]^{-}$ quasi-molecular ion, and the difference between 
Table 3: The 44 biocarta pathways regulated by $P$. hookeri $(p<0.01)$

\begin{tabular}{|c|c|c|c|c|c|}
\hline No. & Pathway & Count & $P$-value & q-value & Gene \\
\hline 1 & MAPK signaling pathway & 10 & $2.42 \mathrm{E}-10$ & 2.15E-09 & $\begin{array}{l}\text { MAPK1;CASP3;EGFR;TGFBR1;MAPK10;MAPK8; } \\
\text { MAPK14;TGFBR2;MAPKAPK2 }\end{array}$ \\
\hline 2 & Adherens junction & 7 & 2.69E-10 & 2.15E-09 & MAPK1;SRC;EGFR;TGFBR1;CSNK2A1;TGFBR2 \\
\hline 3 & Colorectal cancer & 7 & 4.17E-10 & 2.73E-09 & $\begin{array}{l}\text { MAPK1;CASP3;EGFR;TGFBR1;MAPK10;MAPK8; } \\
\text { TGFBR2 }\end{array}$ \\
\hline 4 & Prostate cancer & 7 & $5.81 \mathrm{E}-10$ & 3.49E-09 & MAPK1;EGFR;GSTP1;AR;CDK2 \\
\hline 5 & $\begin{array}{l}\text { Epithelial cell signaling in } \\
\text { Helicobacter pylori infection }\end{array}$ & 6 & $6.58 \mathrm{E}-09$ & 2.96E-08 & SRC;CASP3;EGFR;MAPK10;MAPK8;MAPK14 \\
\hline 6 & Pancreatic cancer & 6 & $8.50 \mathrm{E}-09$ & $3.60 \mathrm{E}-08$ & MAPK1;EGFR;TGFBR1;MAPK10;MAPK8;TGFBR2 \\
\hline 7 & GnRH signaling pathway & 6 & 8.54E-08 & $2.56 \mathrm{E}-07$ & MAPK1;SRC;EGFR;MAPK10;MAPK8;MAPK14 \\
\hline 8 & Focal adhesion & 7 & 2.06E-07 & $5.49 \mathrm{E}-07$ & MAPK1;SRC;EGFR;MAPK10;KDR;MAPK8 \\
\hline 9 & VEGF signaling pathway & 5 & 4.83E-07 & $1.22 \mathrm{E}-06$ & MAPK1;SRC;KDR;MAPK14;MAPKAPK2 \\
\hline 10 & ErbB signaling pathway & 5 & 9.50E-07 & $1.91 \mathrm{E}-06$ & MAPK1;SRC;EGFR;MAPK10;MAPK8 \\
\hline 11 & TGF-beta signaling pathway & 5 & $9.50 \mathrm{E}-07$ & $1.91 \mathrm{E}-06$ & MAPK1;BMP2;TGFBR1;TGFBR2 \\
\hline 12 & Insulin signaling pathway & 5 & 9.19E-06 & $1.32 \mathrm{E}-05$ & MAPK1;MAPK10;MAPK8 \\
\hline 13 & $\begin{array}{l}\text { Complement and } \\
\text { coagulation cascades }\end{array}$ & 4 & 1.19E-05 & $1.52 E-05$ & CFD;CFB;F2 \\
\hline 14 & Purine metabolism & 5 & 1.47E-05 & 1.79E-05 & PDE4B;PDE5A;PNP;NUDT9 \\
\hline 15 & Nitrogen metabolism & 3 & $1.54 \mathrm{E}-05$ & $1.84 \mathrm{E}-05$ & CA2;CA1;CA12 \\
\hline 16 & $\begin{array}{l}\text { Cytokine-cytokine receptor } \\
\text { interaction }\end{array}$ & 6 & $1.56 \mathrm{E}-05$ & 1.84E-05 & BMP2;EGFR;TGFBR1;KDR;TGFBR2 \\
\hline 17 & $\begin{array}{l}\text { Fc epsilon RI signaling } \\
\text { pathway }\end{array}$ & 4 & 2.04E-05 & 2.29E-05 & MAPK1;MAPK10;MAPK8;MAPK14 \\
\hline 18 & Alzheimer's disease & 5 & 3.05E-05 & $3.24 \mathrm{E}-05$ & CASP7;MAPK1;CASP3;BACE1;CDK5R1 \\
\hline 19 & $\begin{array}{l}\text { Toll-like receptor signaling } \\
\text { pathway }\end{array}$ & 4 & 5.57E-05 & $5.42 E-05$ & MAPK1;MAPK10;MAPK8;MAPK14 \\
\hline 20 & Type II diabetes mellitus & $\begin{array}{l}3 \\
3\end{array}$ & $\begin{array}{l}1.05 \mathrm{E}-04 \\
1.61 \mathrm{E}-04\end{array}$ & $\begin{array}{l}9.30 \mathrm{E}-05 \\
129 \mathrm{E}-04\end{array}$ & $\begin{array}{l}\text { MAPK1;MAPK10;MAPK8 } \\
\text { MAPK1:EGFR }\end{array}$ \\
\hline & Starch and sucrose & & & & IVIAR1;EGIR \\
\hline 22 & metabolism & 3 & $1.61 \mathrm{E}-04$ & $1.29 \mathrm{E}-04$ & AMY1B;AMY1A;AMY1C \\
\hline 23 & $\begin{array}{l}\text { Natural killer cell mediated } \\
\text { cytotoxicity }\end{array}$ & 4 & 1.79E-04 & $1.38 \mathrm{E}-04$ & MAPK1;CASP3;ITGAL;ICAM2 \\
\hline 24 & Non-small cell lung cancer & 3 & $1.81 \mathrm{E}-04$ & $1.38 \mathrm{E}-04$ & MAPK1;EGFR \\
\hline 25 & p53 signaling pathway & 3 & $3.73 E-04$ & 2.36E-04 & CASP3;CDK2;CHEK1 \\
\hline 26 & Chronic myeloid leukemia & 3 & 4.77E-04 & 2.83E-04 & MAPK1;TGFBR1;TGFBR2 \\
\hline 27 & Gap junction & 3 & 8.93E-04 & 4.34E-04 & MAPK1;SRC;EGFR \\
\hline 28 & $\begin{array}{l}\text { Regulation of actin } \\
\text { cytoskeleton }\end{array}$ & 4 & 0.001005 & $4.79 E-04$ & MAPK1;EGFR;ITGAL;F2 \\
\hline 29 & $\begin{array}{l}\text { Urea cycle and metabolism } \\
\text { of amino groups }\end{array}$ & 2 & 0.001458 & $6.32 E-04$ & MTAP \\
\hline 30 & Thyroid cancer & 2 & 0.001564 & $6.66 \mathrm{E}-04$ & PPARG;MAPK1 \\
\hline 31 & Cell cycle & 3 & 0.001814 & $7.42 \mathrm{E}-04$ & CDK2;CHEK1 \\
\hline 32 & $\begin{array}{l}\text { Cell adhesion molecules } \\
\text { (CAMs) }\end{array}$ & 3 & 0.002542 & 0.001011 & SELP;ITGAL;ICAM2 \\
\hline 33 & Bladder cancer & 2 & 0.003261 & 0.001255 & MAPK1;EGFR \\
\hline 34 & Wnt signaling pathway & 3 & 0.003625 & 0.001388 & MAPK10;MAPK8;CSNK2A1 \\
\hline 35 & mTOR signaling pathway & 2 & 0.004957 & 0.001844 & MAPK1 \\
\hline 36 & $\begin{array}{l}\text { Amyotrophic lateral sclerosis } \\
\text { (ALS) }\end{array}$ & 2 & 0.005727 & 0.002082 & CASP3;MAPK14 \\
\hline 37 & Hedgehog signaling pathway & 2 & 0.005927 & 0.002144 & BMP2 \\
\hline 38 & Acute myeloid leukemia & 2 & 0.006338 & 0.002259 & MAPK1;PIM1 \\
\hline 39 & Glioma & 2 & 0.007645 & 0.002711 & MAPK1;EGFR \\
\hline 40 & $\begin{array}{l}\text { Adipocytokine signaling } \\
\text { pathway }\end{array}$ & 2 & 0.008105 & 0.002819 & MAPK10;MAPK8 \\
\hline 41 & PPAR signaling pathway & 2 & 0.008818 & 0.003045 & PPARG \\
\hline 42 & $\begin{array}{l}\text { Metabolism of xenobiotics by } \\
\text { cytochrome P450 }\end{array}$ & 2 & 0.008818 & 0.003045 & GSTP1;AKR1C2 \\
\hline 43 & Melanoma & 2 & 0.009062 & 0.003107 & MAPK1;EGFR \\
\hline 44 & $\begin{array}{l}\text { Drug metabolism - } \\
\text { cytochrome P450 }\end{array}$ & 2 & 0.009309 & 0.003169 & GSTP1 \\
\hline
\end{tabular}




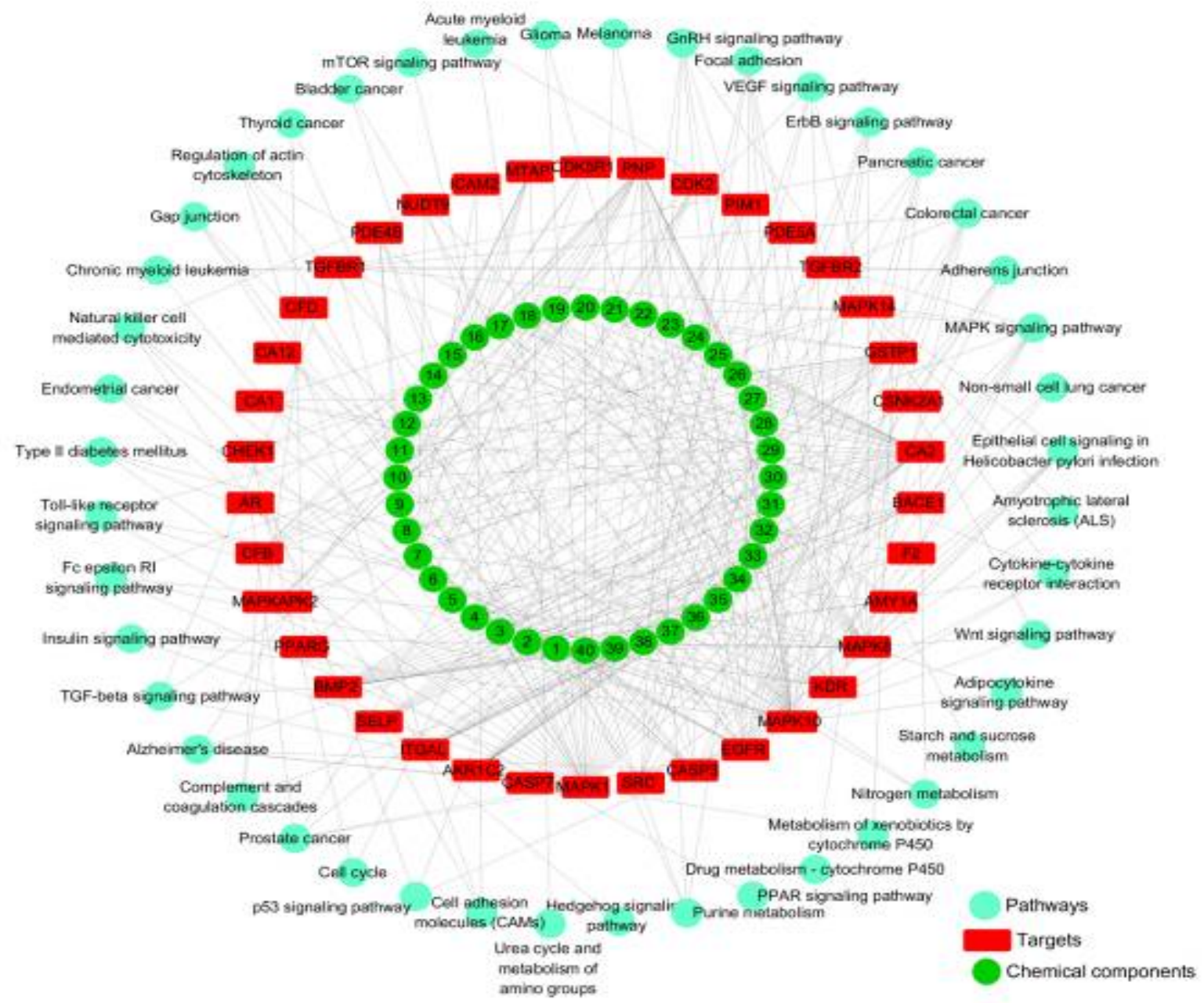

Figure 6: The "component-target-pathway" network of $P$. hookeri

$[\mathrm{M}+\mathrm{Na}]^{+}$and $[\mathrm{M}-\mathrm{H}]^{-}$is $24 \mathrm{Da}$. It can be judged that the ion is a quasi-molecular ion. In the present study, UPLC-Q-TOF/MS technique was used to analyze the chemical components of $P$. hookeri. The results of this study provide a basis for research on the pharmacodynamics and quality control of $P$. hookeri.

It is evident from the results obtained in this study that CA2, MAPK10 and PNP were associated with most of the compounds. Therefore, these factors can be considered to be the main targets. The CA2 gene encodes carbonic anhydrase 2, which is crucial for bone resorption and osteoclast differentiation, and the pathway involved is nitrogen metabolism. There is an imbalance in nitrogen metabolism in patients with RA. However, most patients with RA are in positive nitrogen balance when nitrogen intake is adequate [26]. Purine nucleoside phosphorylase is encoded by PNP gene, and it catalyzes the phosphorolytic cleavage of the $\mathrm{N}$-glycosidic linkage in purine (deoxy) ribonucleosides, with the liberation of free purine base and pentose-1-phosphate. The pathway involved is purine metabolism. Purine metabolism has a highly synergistic effect on immune cell function in RA [27]. The MAPK10 gene encodes mitogen-activated protein kinase 10 , which is associated with inflammation and immune function [28]. The pathways related to RA include MAPK, VEGF, TGF-beta signaling pathway, and focal adhesion. Results from network pharmacological prediction showed that one compound can act on one or more different targets, and one target can also act on different pathways, suggesting that anti-RA property of $P$. hookeri involves multiple components, multiple targets, and multiple pathways.

The network pharmacology method used in this study is an innovative methodology due to the establishment of multilayer networks of disease target-drug for forecasting drug targets in an integral manner, and for boosting efficient drug discovery [29]. This method represents a breakthrough, when compared with the traditional herbal medicine research methodology 
of gene-target-disease, and initiates a new system of multiple genes-multiple targetscomplex diseases.

This is the first study on the mechanism involved in the anti-RA effect of $P$. hookeri, and the first to use the novel technique of network pharmacology. Using this method, the main targets and pathways were successfully predicted, thereby providing a foundation for further research. This method is important for the study of complex drugs and should be applied in future studies.

\section{CONCLUSION}

A UPLC-Q-TOF/MS method has been successfully developed for the rapid analysis of chemical components of $P$. hookeri. A total of 40 compounds have been identified. Network pharmacology method enables the prediction of the therapeutic effect of $P$. hookeri on RA via a mechanism involving multiple components, multiple targets and multiple pathways.

\section{DECLARATIONS}

\section{Acknowledgement}

The authors gratefully acknowledge the financial support from National Natural Science Foundation of China (no. 81274193), and Construction Plan of Scientific Research Innovation Team in Sichuan Province (no. 11TD004).

\section{Conflict of interest}

The authors declare that no conflict of interest is associated with this work.

\section{Contribution of authors}

The authors declare that this work was done by the authors named in this article and all liabilities pertaining to claims relating to the content of this article will be borne by them. Ce Tang and Yi Zhang conceived and designed the experiments; Ce Tang, Hai-Jiao Li, Gang Fan, and Ting-Ting Kuang performed the experiments; Ce Tang and Yi Zhang wrote the paper; Ce Tang, Ting-Ting Kuang, Xian-Li Meng, and Zhong-Mei Zou analyzed the data. All authors reviewed the manuscript.

\section{REFERENCES}

1. Maijer KI, Gudmann NS, Karsdal MA, Gerlag DM, Tak
PP, Bay-Jensen AC. Neo-Epitopes-Fragments of Cartilage and Connective Tissue Degradation in Early Rheumatoid Arthritis and Unclassified Arthritis. Plos One 2016; 11(3): e0149329.

2. Yang J, Zuo F, Wei ZC, Zeng $Y$, Meng XL, Zhang $Y$. Therapeutic Effects and Mechanisms of Total Glucosides of Herba Pterocephali on Adjuvant Arthritis Rats. Tradit Chin Drug Res Pharmaco 2016; 27(6): 788794.

3. Yangben ZX, Pengmao DZ, Xin CJ, Zhuoma CD, Gong B. Clinical Evaluation of Tibetan Medicine in Treating Rheumatoid Arthritis. J Med Pharm Chin Minorities 2013; 5: 18-19.

4. Ri S, Zhou XB. Clinical observation of 101 cases of rheumatoid arthritis treated by Tibetan medicine. J Med Pharm Chin Minorities 2017; 12: 36-37.

5. Tang C, Fan G, Li Q, Su JS, Meng XL, Zhang $Y$. Simultaneous determination of ten compounds in two main medicinal plant parts of Tibetan herb, Pterocephalus hookeri (CB Clarke) Höeck, by ultra-high performance liquid chromatography-photodiode array. Trop J Pharm Res 2017; 16 (6): 1407-1416.

6. Tian J, Wu FE, Qiu MH, Nie RL. Triterpenoid saponins from Pterocephalus hookeri. Phytochem 1993; 32 (6): 1535-1538.

7. Wang J, Zhao $K H$, Geng ZJ, Lai XR, Yang WJ, Hu XX, Zhang $Y$, Jiangrong SL. Comparison Study on Zhenbu Disease of Tibetan Medicine and Bi Syndrome in Traditional Chinese Medicine. Modern Tradit Chin Med Mater Med-World Sci Technol 2015; 17(10): 2167-2171.

8. Zhang L, Hu JJ, Lin JW, Fang WS, Du GH. Antiinflammatory and analgesic effects of ethanol and aqueous extracts of Pterocephalus hookeri (C.B. Clarke) Höeck. J Ethnopharmacol 2009; 123 (3): 510-514.

9. Shen XF, Zeng Y, Li JC, Tang C, Zhang Y, Meng XL. The anti-arthritic activity of total glycosides from Pterocephalus hookeri, a traditional Tibetan herbal medicine. Pharm Biol 2017; 55(1): 560-570.

10. Wu L, Wang $Y$, Nie J, Fan $X$, Cheng $Y$. A network pharmacology approach to evaluating the efficacy of Chinese medicine using genome-wide transcriptional expression data. Evid Based Complement Alternat Med 2013; 2013: 915343.

11. Zhang JF, Huang S, Shan LH, Chen L, Zhang $Y$, Zhou $X L$. New Iridoid Glucoside from Pterocephalus hookeri. J Ogr Chem. 2015; 35: 2441-2444.

12. Huang S, Zhang JF, Shan LH, Zhang Y, Zhou XL. A novel tetrairidoid glucoside from Pterocephalus hookeri. Heterocycles 2017; 94(3): 485.

13. Qi LW, Chen CY, Li P. Structural characterization and identification of iridoid glycosides, saponins, phenolic acids and flavonoids in Flos Lonicerae Japonicae by a fast liquid chromatography method with diode-array detection and time-of-flight mass spectrometry. Rapid Commun Mass Spectrom 2009; 23(19): 3227-3242.

14. Sun $H Y$, Liu $M X$, Lin $Z T$, Jiang $H X$, Niu $Y Y$, Wang $H$, Chen SZ. Comprehensive identification of 125 multifarious constituents in Shuang-huang-lian powder

Trop J Pharm Res, June 2018; 17(6): 1109 
injection by HPLC-DAD-ESI-IT-TOF-MS. J Pharm Biomed Anal 2015; 115: 86-106.

15. Ling $Y$, Liu $K Y$, Zhang $Q$, Liao L, Lu $Y H$. High performance liquid chromatography coupled to electrospray ionization and quadrupole time-of-flightmass spectrometry as a powerful analytical strategy for systematic analysis and improved characterization of the major bioactive constituents from Radix Dipsaci. $J$ Pharm Biomed Anal 2014; 98: 120-129.

16. Tian $X Y$, Wang $Y H$, Liu HY, Yu SS, Fang WS. On the Chemical Constituents of Dipsacus asper. Chem Pharm Bull 2007; 55(12): 1677-1681.

17. Prasad $D$, Juyal V, Singh $R$, Singh V, Pant $G$, Rawat MS. A new secoiridoid glycoside from Lonicera angustifolia. Fitoterapia 2000; 71(4): 420-424.

18. Wu YC, Yin YJ, Li YM, Guo FJ, Zhu GF. Secoiridoid/iridoid subtype bis-iridoids from Pterocephalus hookeri. Maqn Reson Chem 2014; 52(11): 734-738.

19. Gülcemal D, Masullo $M$, Alankuş-Calişkan O, Karayildirim T, Senol SG, Piacente S, Bedir E. Monoterpenoid glucoindole alkaloids and iridoids from Pterocephalus pinardii. Maqn Reson Chem 2010; 48(3): 239-243.

20. Tian J, Wu FE, Qiu MH, Nie RL. Triterpenoid saponins from Pterocephalus hookeri. Phytochem 1993; 32 (6): 1535-1538.

21. Zhang $Y$, Li WJ, Meng $X L$, Shen $P$, Jia MR. Chemical Constituents of Tibet Drug Pterocephalus hookeri (C. B. Clarke) Hoeck. J Chengdu Univ Tradit Chin Med 2002; 25(3): 41-42.

22. Tian J, Wu FE, Qiu MH, Nie RL. Two triterpenoid saponins from Pterocephalus bretschneidri. Phytochem 1993; 32(6): 1539-1542.

23. Tang C, Su JS, Yang J, Zuo F, Meng XL, Zou ZM, Zhang $Y$. Content Determination of Oleanolic Acid and Ursolic Acid from Different Medicinal Parts in Tibetan Medicine
Pterocephalus hookeri by UPLC-PDA. J China Pharm 2017; 28(7): 929-932.

24. Guo CX, Wu YC, Zhu YZ, Wang YC, Tian LL, Lu Y, Han C, Zhu GF. In Vitro and In Vivo Antitumor Effects of nButanol Extracts of Pterocephalus hookeri on Hep3B Cancer Cell. Evid Based Complement Alternat Med 2015; 2015: 159132.

25. Zuo F, Wei $T$, Tong $D$, Zhang $Y$, Chen XR, Zeng $Y$. Pharmacodynamic mechanism of modified Ganlu Yaoyu San in treatment of rheumatoid arthritis based on MAPK signaling pathway. Chin J Chin Mater Med 2017; 42(7): 1245-1250.

26. Clark WS, Bauer W, Appleton J, Manning E. The Relationship of Alterations in Mineral and Nitrogen Metabolism to Disease Activity in a Patient. Acta Rheumatol Scand 1956; 2(4): 193-212.

27. Morgan SL, Oster RA, Lee JY, Alarcón GS, Baggott JE. The effect of folic acid and folinic acid supplements on purine metabolism in methotrexate-treated rheumatoid arthritis. Arthritis Rheum 2004; 50(10): 3104-3111.

28. Vargas-Alarcón G, Posadas-Romero C, Villarreal-Molina T, Alvarez-León E, Angeles-Martinez J, PosadasSanchez R, Monroy-Muñoz I, Luna-Fuentes S, González-Salazar C, Ramirez-Bello J, et al. IL-24 gene polymorphisms are associated with cardiometabolic parameters and cardiovascular risk factors but not with premature coronary artery disease: the genetics of atherosclerotic disease Mexican study. J Interferon Cytokine Res 2014; 34(9): 659-666.

29. Ma $X H$, Lv B, Li $P$, Jiang $X Q$, Zhou $Q$, Wang $X Y$, Gao $X M$. Identification of "Multiple Components-Multiple Targets-Multiple Pathways" Associated with Naoxintong Capsule in the Treatment of Heart Diseases Using UPLC/Q-TOF-MS and Network Pharmacology. Evid Based Complement Alternat Med 2016; 2016: 9468087. 